\title{
TRANSFORMAÇÕES DE REPRESENTAÇÕES VISUAIS DE MÚLTIPLOS E DIVISORES DE UM NÚMERO
}

\author{
TRANSFORMATIONS OF VISUAL REPRESENTATIONS \\ OF MULTIPLES AND DIVISORS OF A NUMBER
}

\section{CAMBIO DE REPRESENTACIONES VISUALES DE LOS MÚLTIPLOS Y DIVISORES DE UN NÚMERO}

\author{
Paula Montenegro ${ }^{\mathrm{I}}$ \\ Cecília Costa ${ }^{\mathrm{II}}$ \\ BERNARDINO LOPES ${ }^{\text {III }}$
}

\begin{abstract}
Resumo A resolução de problemas é uma das capacidades matemáticas fundamentais e uma base para a aprendizagem de conceitos, procedimentos matemáticos e representações. Não obstante, os alunos evidenciam dificuldades na resolução de problemas e na multiplicidade de representações para um dado conceito matemático. Este estudo procurou sistematizar a relevância e a eficácia da introdução frequente de representações visuais na lecionação dos conteúdos relativos às noções de múltiplo e divisor de um número natural, e do seu impacto na resolução de problemas com essas noções. Segue uma metodologia qualitativa analisando uma intervenção de ensino num grupo de 17 alunos (10-11 anos de idade). Os resultados mostraram que (i) as representações visuais permitiram transformações de representações (tratamentos e conversões), como as representações simbólicas e verbais, (ii) a introdução de representações visuais proporcionou um ambiente de aprendizagem com representações múltiplas, com uma maior variedade de representações na resolução de problemas, e (iii) esse efeito prolongou-se no intervalo de tempo considerado. Concluímos que a utilização frequente de representações visuais potenciou as transformações de representações tendo como consequência uma melhoria no desempenho geral dos alunos.
\end{abstract}

Palavras-chave: Divisores; Múltiplos; RepresentaÇões visuais; Resolução de problemas.

\footnotetext{
IUniversidade de Trás-os-Montes e Alto Douro (UTAD). Vila Real - Portugal.

"Universidade de Trás-os-Montes e Alto Douro (UTAD). Vila Real - Portugal.

IIIUniversidade de Trás-os-Montes e Alto Douro (UTAD). Vila Real - Portugal.
} 
Abstract Problem solving is one of the most important mathematical capacities and bases for learning of mathematical concepts, procedures and representations. However, the students show difficulties in problem solving and in a multiplicity of representations of a given mathematical concept/object. The aim of this study is to determine the relevance and the impact of using visual representations and their transformations in the teaching process of divisors and multiple of a number, and their impact in problem solving with these notions. It follows a qualitative methodology and in this paper we analyze an educational intervention in a group of 17 students (10-11 years old). The results show that (i) visual representations allowed transformations of representations (treatments and conversions) with the same characteristics of the symbolic and verbal representations (ii) these visual representations provided a learning environment with multiple representations that diversified the transformation of representations (treatments and conversions), and (iii) this effect was extended in the considered interval of time (three months). We conclude that the use of visual representations enhances the transformations of representations which contributed to the improvement of the students overall performance.

Key-words: Divisors; Multiples; Problem solving; Visual representations.

RESUMEN La resolución de problemas é una das capacidades matemáticas fundamentales y una base para el aprendizaje de conceptos matemáticos, procedimientos y representaciones. No obstante, los estudiantes muestran dificultades en la resolución de problemas y en la multiplicidad de representaciones para un dado concepto matemático. Este trabajo trató de sistematizar la relevancia y la importancia de la introducción frecuente de representaciones visuales en la enseñanza de los contenidos relativos a nociones de múltiple y divisor de un número natural, y del su impacte en la resolución de problemas. En este estudio se sigue la metodología cualitativa y se analiza una intervención educativa en un grupo de 17 estudiantes (10-11 años). Los resultados muestran que (i) las representaciones visuales permite transformaciones (tratamientos y conversiones), iguales a transformaciones de las representaciones simbólicas y verbales, (ii) esta intervención proporcionó un ambiente de aprendizaje con múltiples representaciones que conllevó a una diversificación de la transformación de representaciones en la resolución de problemas y (iii) este efecto se prolongó en el intervalo de tiempo considerado. Se concluyó que el uso de representaciones visuales potencia las transformaciones y contribuyó para mejorar el rendimiento general de los estudiantes.

Palabras clave: Divisores, Múltiples - Representaciones visuales - Resolución de problemas.

\section{INTRODUÇ̃̃o}

A resolução de problemas é uma das capacidades matemáticas fundamentais e uma base para a aprendizagem de conceitos, procedimentos matemáticos e representações. Os alunos evidenciam dificuldades na resolução de problemas e na multiplicidade de representações para um dado conceito/objeto matemático. Spiro (1988) destaca a capacidade de construir e alternar múltiplas perspectivas como essencial para o sucesso da aprendizagem; Dienes (1973) sugere a utilização de representações diferentes do mesmo objeto e argumenta que os mesmos conceitos representados de maneiras distintas fornecem aos alunos oportunida- 
de de abstração o que, segundo Bransford e Schwartz (1999), aumenta a probabilidade de transferência para novas situações. As formas mais comuns de representações utilizadas nos enunciados de situações problemáticas, e na sua resolução, são palavras (linguagem verbal), figuras, tabelas, gráficos, esquemas (linguagem visual) e símbolos numéricos ou algébricos (linguagem simbólica). Essas representações verbais, visuais e simbólicas pertencem a sistemas de linguagem diferentes com códigos e regras específicos (Calado, 1995). A linguagem verbal e a linguagem simbólica são amplamente reconhecidas na atividade matemática (Barbosa, 2010; Duval, 2006a), mas a linguagem visual, embora seja reconhecido o seu valor pedagógico, nem todos os professores e investigadores em Educação Matemática lhe dão o mesmo estatuto (Dreyfus, 1991). Neste trabalho, temos por objetivo contribuir para a discussão mostrando que a utilização frequente de representações visuais no ensino pode melhorar o desempenho dos alunos na resolução de problemas. Em particular, no domínio dos números e operações, elucidamos o papel importante que as representações visuais tiveram no desempenho de um grupo de alunos na resolução de problemas envolvendo as noções de máximo divisor comum (m.d.c.) e de mínimo múltiplo comum (m.m.c.).

\section{Problema de investigação}

A deficiente proficiência dos alunos portugueses do ensino básico na resolução de problemas é frequente em estudos de avaliação nacionais e internacionais (GAVE, PISA) e em trabalhos de investigação (por exemplo, Barbosa 2010; Luís, 2015). O Programa de Matemática em vigor (MEC, 2013) destaca a importância da resolução de problemas como uma das capacidades matemáticas fundamentais e uma base para a aprendizagem de conceitos, procedimentos matemáticos e representações. Consideramos representações quaisquer configurações que possam substituir uma entidade de qualquer forma (Goldin, 2008). O NCTM (2000) reconhece que o termo representação se refere e se aplica tanto ao produto como ao processo obtido tanto externamente, como ao que ocorre na mente dos estudantes quando fazem matemática. Nesse contexto, é pertinente associar ao processo o conceito de visualização e, a um dos seus produtos, o conceito de representação visual. Os dois conceitos têm sido alvo de diversas interpretações não havendo ainda consenso para uma definição formal. Arcavi (2003) e Sedig e Sumner (2006), parafraseando outros autores, definem, respectivamente, o conceito de visualização e o de representação visual. Visualização é

\footnotetext{
“... capacidade, o processo e o produto da criação, interpretação, uso e reflexão sobre figuras, imagens, diagramas, nas nossas mentes, no papel ou com ferramentas tecnológicas, com a finalidade de retratar e comunicar informação, pensar e desenvolver previamente ideias desconhecidas e entendimentos avançados" (ARCAVI, 2003). ${ }^{1}$
}

Texto original: "the ability, the process and the product of creation, interpretation, use of and reflection upon pictures, images, diagrams, in our minds, on paper or with technological tools, with the purpose of depicting and communicating information, thinking about and developing previously unknown ideas and advancing understandings" (ARCAVI, 2003, p. 217). 
Uma representação visual é "uma coleção de símbolos gráficos que codificam visualmente as propriedades causais, funcionais, estruturais e semânticas e as relações de um mundo representado - seja abstrato ou concreto" (SEDIG \& SUMNER, 2006). ${ }^{2}$ Essas definições são abrangentes e incluem representações visuais que podem apresentar símbolos verbais e/ou simbólicos, desde que ligadas à compreensão da matemática (processo ou produto) e se cumprirem o requisito inerente às suas definições. Vários autores reconhecem a interação entre a visualização e a didática da matemática (por exemplo El Mouhayar e Jurdak, 2013; Presmeg, 2006; Stylianou e Silver, 2004), identificando uma função de ponte das representações visuais entre as representações simbólicas e as verbais (Presmeg, 2006); ou como facilitadoras da comunicação dentro da sala de aula (Ryve, Nilsson \& Pettersson, 2013). Mas, apesar desse reconhecimento, autores vários veem dificuldades, por exemplo, Van Garderen (2006) as reconhece no uso de diagramas na resolução de problemas por uma parte significativa de alunos, mesmo depois de conhecerem o procedimento para a sua construção, o que leva alguns autores (por exemplo, Ainsworth, 2008) a defenderem o uso de regras, pois a aprendizagem de conceitos científicos com várias representações torna-se, ela própria, complexa. De acordo com Duval (2006b), só existe compreensão dos objetos matemáticos quando somos capazes de os representar pelo menos de dois modos diferentes, transformando-os entre si, o que nos leva a analisar o efeito na aprendizagem de situações de ensino que valorizem a transformação de representações, em particular de representações visuais. Duval (2006a; 2006b) identifica dois tipos de transformações de representações: Tratamentos e conversões. Parafraseando o trabalho de Duval (2006a; 2006b), tratamentos são transformações que ocorrem dentro do mesmo sistema de linguagem, como simplificar uma expressão numérica ou desenhar simetrias numa figura; conversões consistem na transformação de uma representação de um sistema para outro, como representar uma fração (linguagem simbólica) numa figura (linguagem visual). Da conversão entre representações se potenciará a abstração (Ainsworth, 2008; Dienes, 1973; Duval, 2006a; 2006b; Spiro, 1988), processo esse que levará os alunos a criar as entidades mentais que servem de base para novos procedimentos e conceitos, num nível mais elevado de organização do seu conhecimento e raciocínio. Assim, temos por objetivo averiguar o efeito da utilização de representações visuais em conjunto com representações verbais e simbólicas, na aprendizagem das noções de múltiplo e de divisor de um número natural. Para o efeito elaboramos a seguinte questão de investigação: Qual o impacto da introdução de representações visuais, e suas transformações, na resolução de problemas com múltiplos e divisores?

\section{Metodologia}

Este trabalho teve a pretensão de compreender melhor a particularidade da introdução da exploração frequente de representações visuais na resolução de exercícios e problemas com as noções de múltiplo e divisor de um número natural. Optou-se por uma metodologia qualitativa (Cohen, Manion \& Morrison, 2007), pois pretende-se conhecer o impacto da

2 Texto original: "a collection of graphical symbols that visually encode causal, functional, structural, and semantic properties and relationships of a represented world - either abstract or concrete" (SEDIG \& SUMNER, 2006, p. 2).

58 Comunicações |Piracicaba | v. 24 | n. 1 | p. 55-68 | janeiro-abril 2017 
utilização de representações visuais em simultâneo com representações simbólicas e verbais no ensino e aprendizagem dos conceitos envolvidos.

\section{INTERVENIENTES E MATERIAIS}

Os alunos participantes neste estudo beneficiaram de uma intervenção de ensino (36 aulas) ancorada na utilização frequente de representações visuais no ensino e exploração dos conteúdos envolvidos. Por exemplo, foram empregados diagramas de Venn na lecionação e exploração dos divisores de um número natural ou a reta numérica para os múltiplos, a par de representações simbólicas e verbais e outras representações visuais. É nossa convicção que um ensino com representações múltiplas potencia a compreensão dos assuntos matemáticos, o que provavelmente terá influência no tempo de retenção desses assuntos na memória dos aprendentes. Pretendemos verificar se as representações visuais têm, ou não, um papel nessa influência. Essa aferição baseou-se nas respostas a um teste (Figura 1) aplicado em dois momentos distintos: o primeiro imediatamente a seguir à lecionação dos domínios em questão, no dia 23 de novembro do ano, e o segundo no último dia do mês de fevereiro do ano seguinte, pois havia garantia que, nesse intervalo de tempo não havia repetição dos conceitos envolvidos, o que não se podia garantir depois dessa data.

Figura 1 - Teste aplicado.

1) Escreva os divisores de 9.

2) Represente de duas formas diferentes os múltiplos naturais de 5.

3) Calcule os divisores comuns de 16 e 18. Qual o seu máximo divisor comum?

4) Calcule o mínimo múltiplo comum de 4 e 5.

5) Os números 10 e 15 são números primos entre si? Justifique.

"Preparando a festa de aniversário I"

6) O Francisco quer organizar a sua festa de aniversário e pretende oferecer aos seus convidados uns sacos com berlindes e bonecos "Mínimos". Cada saco deverá ter o mesmo conteúdo e os objetos não podem sobrar. A mãe do Francisco the comprou 18 bonecos e 42 berlindes.

a) Qual o número máximo de sacos que o Francisco pode fazer?

b) Qual o conteúdo de cada saco?

Explique como chegou à sua resposta.

"Preparando a festa de aniversário II"

7) Para enfeitar a sala onde vai receber os seus convidados, Francisco utilizou uma gambiarra com luzes de duas cores: vermelho e azul. Sabendo que as luzes de cor vermelha acendem a cada 6 segundos e as de cor azul acendem a cada 8 segundos e que as luzes acenderam juntas às $16 \mathrm{~h} 30 \mathrm{~min}$, a que horas acenderão novamente as luzes ao mesmo tempo? Explique como chegou à sua resposta.

Entre os dois momentos de aplicação do teste, lecionaram-se conteúdos Retas e ângulos e Números racionais não negativos (MEC, 2013), diversificando também as representações dentro dos três sistemas de linguagem, com recurso a representações visuais adequadas aos novos conceitos matemáticos. A resolução do teste, nos dois momentos de 
aplicação, teve a duração de 30 minutos. Os dados foram recolhidos dos cadernos diários dos alunos, da observação participante da professora, e dos resultados dos testes referidos. $\mathrm{Na}$ análise dos registos dos cadernos diários, começamos por identificar as representações, e respectivas transformações, utilizadas durante a intervenção de ensino. Para as representações foram criadas duas categorias de análise: Representações e Transformações de representações (Tabela 1). Para a primeira categoria formaram-se ainda subcategorias em (i) linguagem visual: representações figurativas, em diagrama de Venn, reta numérica, em esquema e em tabela; (ii) para as representações em linguagem simbólica as numéricas e, finalmente (iii) a linguagem natural para as representações em linguagem verbal. No que diz respeito às Transformações de representações, consideramos as Conversões e os Tratamentos. Nos últimos, distinguimos duas subcategorias conforme altere $\left(T_{R \rightarrow R}\right)$, ou não $\left(T_{R}\right)$, o sistema de representação dominante.

Tabela 1 - Categorias, subcategorias e códigos respetcivos.

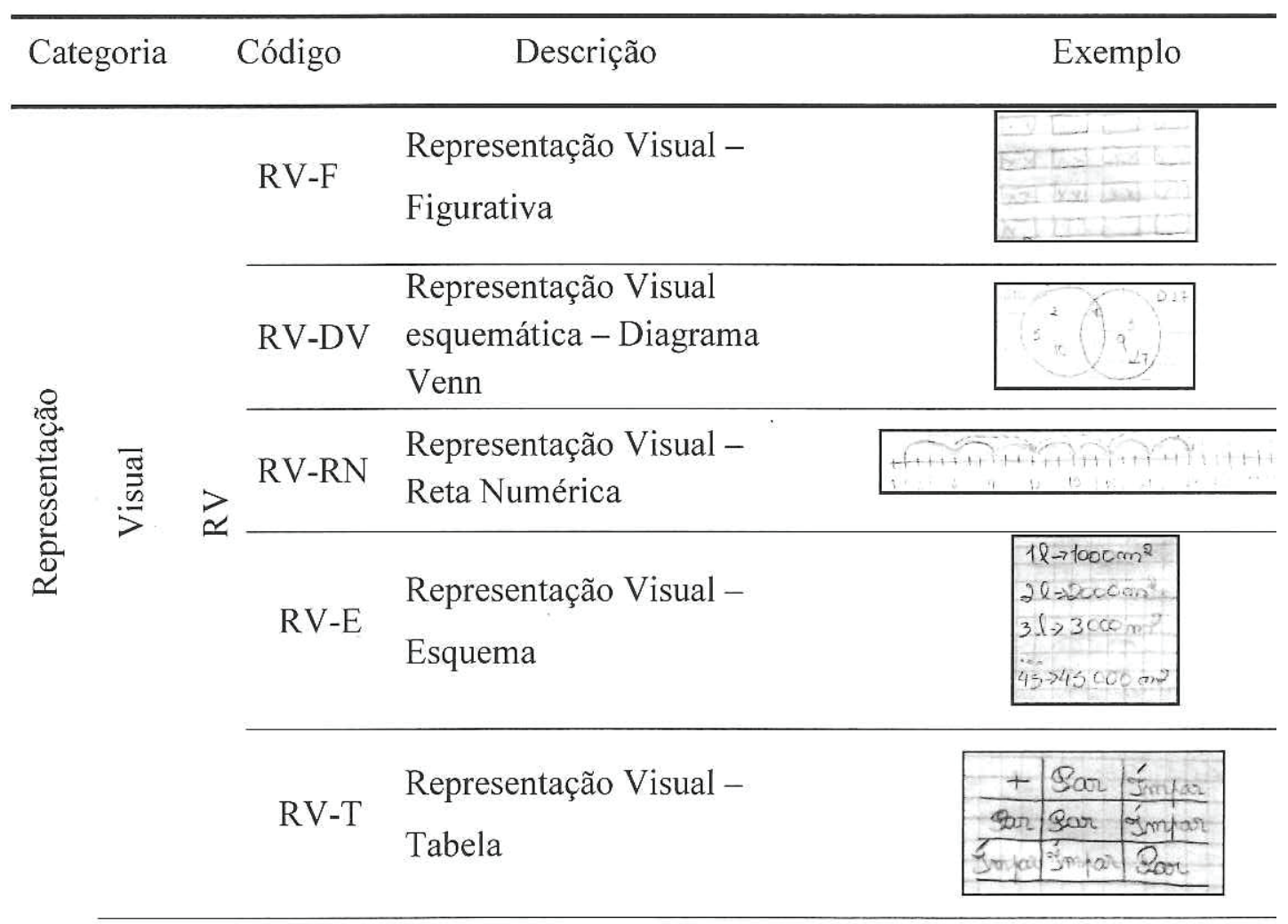




\begin{tabular}{|c|c|c|c|c|}
\hline \multicolumn{2}{|c|}{ Categoria } & Código & Descrição & Exemplo \\
\hline & 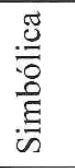 & RS-N & $\begin{array}{l}\text { Representação } \\
\text { Simbólica - Numérica }\end{array}$ & $a=25+19=44$ \\
\hline & 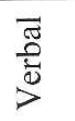 & RLN & $\begin{array}{l}\text { Representação em } \\
\text { Linguagem Natural }\end{array}$ & $\begin{array}{l}\text { Proprudade ermutatura: Sodemes tro- } \\
\text { earta ordom dar forelas que o resul- } \\
\text { tado mâ se altot }\end{array}$ \\
\hline & ? & $\mathrm{T}_{\mathrm{R}}$ & $\begin{array}{l}\text { Tratamento numa } \\
\text { representação sem } \\
\text { alteração do sistema de } \\
\text { representação dominante }\end{array}$ & 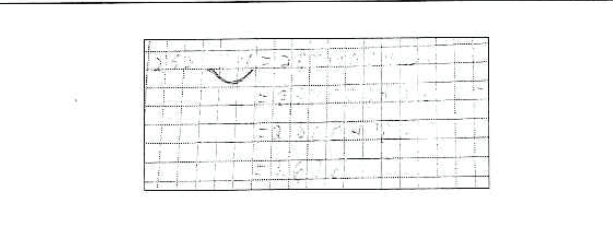 \\
\hline 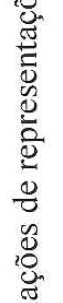 & 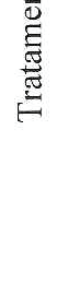 & $\mathrm{T}_{\mathrm{R} \rightarrow \mathrm{R}}$ & $\begin{array}{l}\text { Tratamento numa } \\
\text { representação com } \\
\text { alteração do sistema de } \\
\text { representação dominante }\end{array}$ & $\begin{array}{l}\begin{array}{c}23 \times 15-60 \text { y } \\
=45-15= \\
30 c\end{array}=\begin{array}{l}\text { A utilização de pequenos } \\
\text { traços evidenciou a } \\
\text { prioridade das operações, }\end{array} \\
\text { transformando a representação numérica } \\
\text { numa visual. }\end{array}$ \\
\hline$\frac{\overbrace{0}^{0}}{\tilde{D}^{\pi}}$ & $\begin{array}{l}0 \\
0 \\
0 \\
00 \\
0 \\
0 \\
0\end{array}$ & $R \stackrel{C}{\rightarrow} R$ & $\begin{array}{l}\text { Conversão de } \\
\text { representações entre } \\
\text { sistemas de linguagem }\end{array}$ & $\begin{array}{l}\frac{0+8}{2} \rightarrow 2 \text { dirde a soma de to lom } 8 \\
\text { Este exemplo mostra uma conversão de } \\
\text { uma representação em linguagem } \\
\text { simbólica para uma representação em } \\
\text { linguagem verbal (RS-N } \stackrel{C}{\rightarrow} \text { RLN). }\end{array}$ \\
\hline
\end{tabular}

Para a análise das respostas do teste contabilizaram-se as respostas corretas e as parcialmente corretas com evidências de chegar à resposta correta.

\section{Resultados}

\section{Resultantes da intervenção de ensino}

Em todas as aulas usaram-se representações em linguagem verbal e simbólica. Utilizaram-se representações visuais de diferentes tipos: figurativas, diagrama de Venn, reta numérica, esquema e tabela. Nenhuma dessas representações teve a frequência das representações em linguagem verbal e simbólica, apesar de se ter usado em todas as aulas algum tipo de representação visual.

A título de exemplo, mostra-se na Figura 2 algumas das representações usadas durante as aulas para a exploração dos divisores de um número natural. 
Figura 2 - Exemplo de uma página do caderno diário de um dos alunos participantes.

Inspeção dos divisores de 6 (RS-N) seguida de tratamento visual à representação numérica $\left(\mathrm{T}_{\mathrm{RS}-\mathrm{N} \text { ? } \mathrm{RV}}\right)$ alterando o sistema de renresentacão de numérico nara visual.

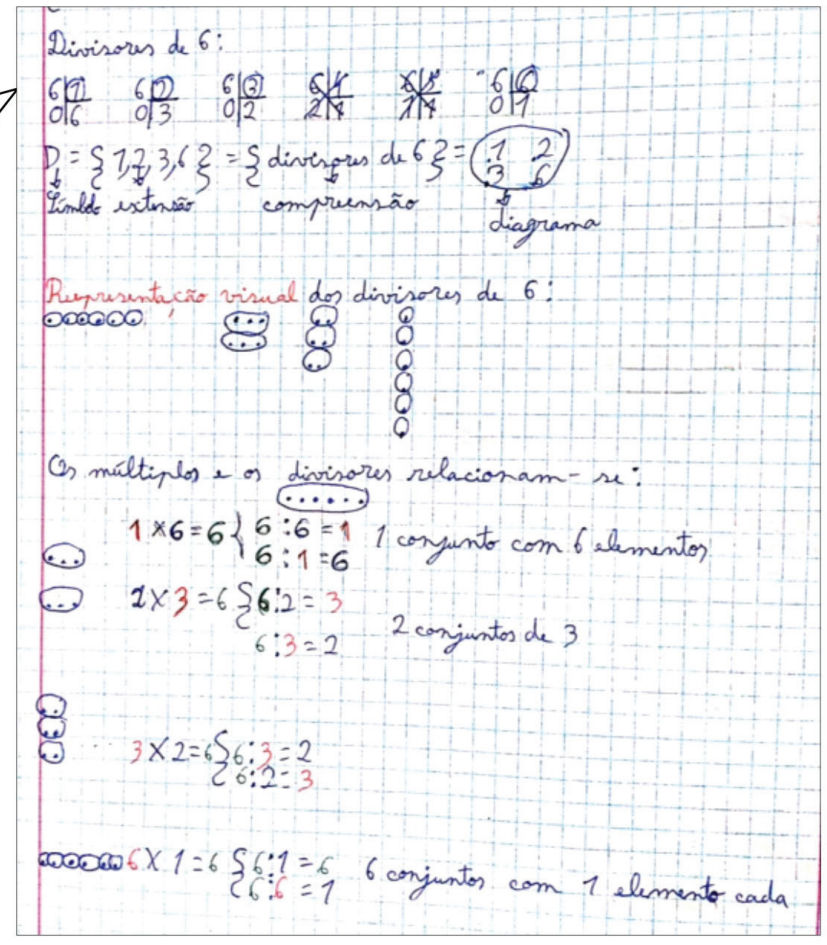

Representação numérica (RS-N), verbal (RLN) e visual (RV-D) dos divisores de 6.

Representação figurativa dos divisores de 6

(RV-F)

Conversão dos divisores de 6 de representação em linguagem visual para simbólica e verbal:

(RV-F RS-N RLN).

Tratamento visual dos divisores de 6 , com alteração do sistema de representação dominante de simbólico para visual ( $\mathrm{T}_{\mathrm{RS}}$ $\mathrm{N}$ ? RV) 
Nessa aula, procedeu-se à inspeção dos divisores de 6, de acordo com a sua definição, com recurso ao algoritmo da divisão, da sua representação segundo a teoria de Conjuntos: símbolo e conjunto escrito em extensão (linguagem simbólica), em compreensão (linguagem verbal) e representações visuais: diagrama e por fim, explorou-se a sua representação figurativa (linguagem visual).

Com a introdução dessa representação, relacionaram-se as noções de múltiplo e divisor, procedendo-se ao seu tratamento e conversão para linguagem natural e para representação simbólica. Depois, fizeram-se tratamentos e conversões. Iniciou-se por um tratamento na representação numérica dos divisores de seis no algoritmo da divisão, realizado pelos alunos; seguiu-se a sua conversão para outros tipos de representação conhecidos (linguagem natural e diagrama), procurando-se uma representação visual para o mesmo objeto matemático.

Em seguida, relacionaram-se os conceitos de múltiplo e divisor de um número, fazendo-se conversões simultâneas da representação visual para a representação numérica e para a linguagem natural.

Fizeram-se tratamentos visuais para evidenciar determinadas relações, isto é, tratamentos a representações em determinado sistema de representação com o intuito de destacar algo por meio de adição de cor, por exemplo, alterando o sistema de representação dominante.

Durante a intervenção de ensino, foram efetuados tratamentos às representações em linguagem natural, às representações simbólicas (numéricas) e às representações visuais (diagrama de Venn, representações figurativas, à reta numérica, esquema e tabela).

Os tratamentos mais comuns feitos às representações numéricas alteraram o sistema de representação dominante para um sistema visual, apesar de a representação de base continuar no mesmo sistema (Tabela 2, à esquerda e embaixo). Esse tipo de tratamento foi efetuado, durante o processo de visualização, transformando a representação inicial numa representação visual.

No que concerne às conversões, registraram-se conversões de representações: (i) da representação inicial em linguagem verbal para a representação final em linguagem visual ; (ii) da representação inicial em linguagem visual para a representação final em linguagem verbal ; e (iii) da representação inicial em linguagem visual para a representação final em linguagem simbólica (Tabela 2, à direita). 
Tabela 2 - Exemplos de transformações de representações efetuadas durante as aulas

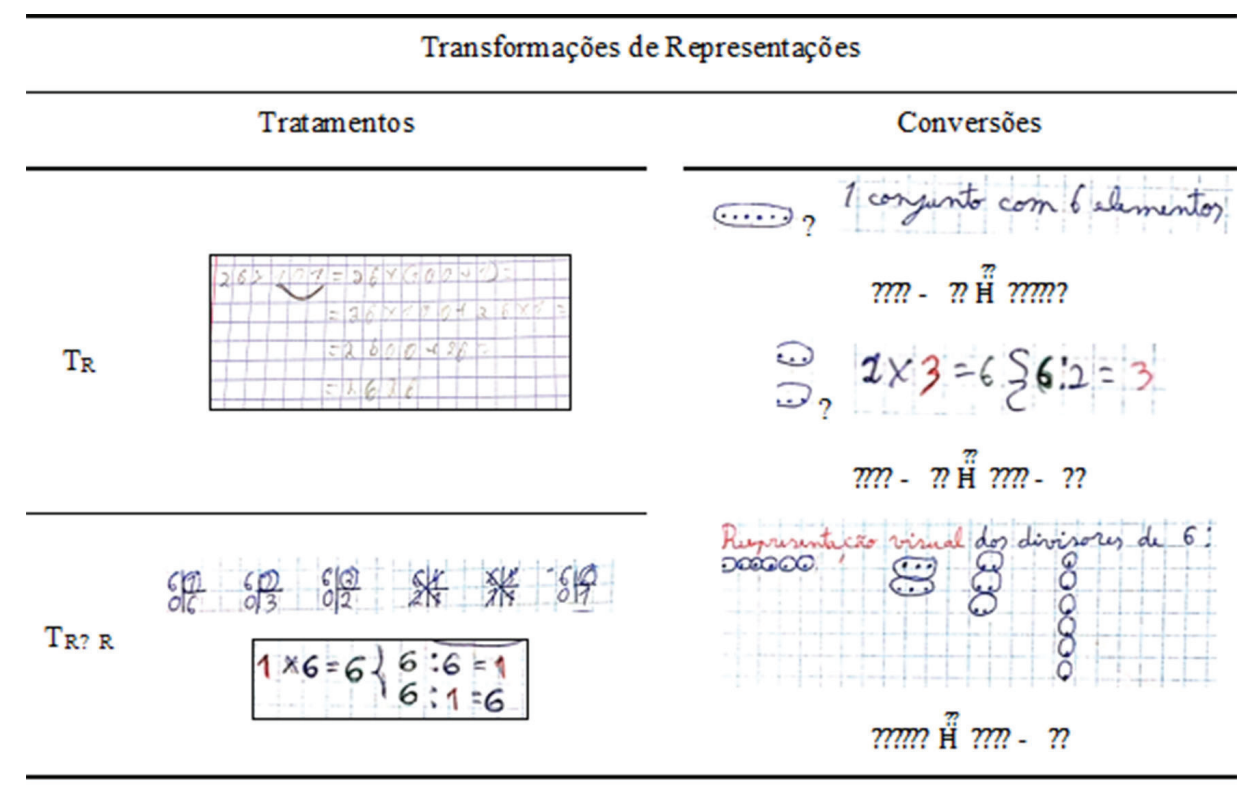

Na resolução de problemas também constatou-se a realização de tratamentos e conversões de representações nos diferentes sistemas de linguagem (Figura 3).

Figura 3 - Tratamentos e conversões resultantes da discussão coletiva depois de resolvido o problema (em cima) e exemplificação com outra situação mais simples (embaixo).

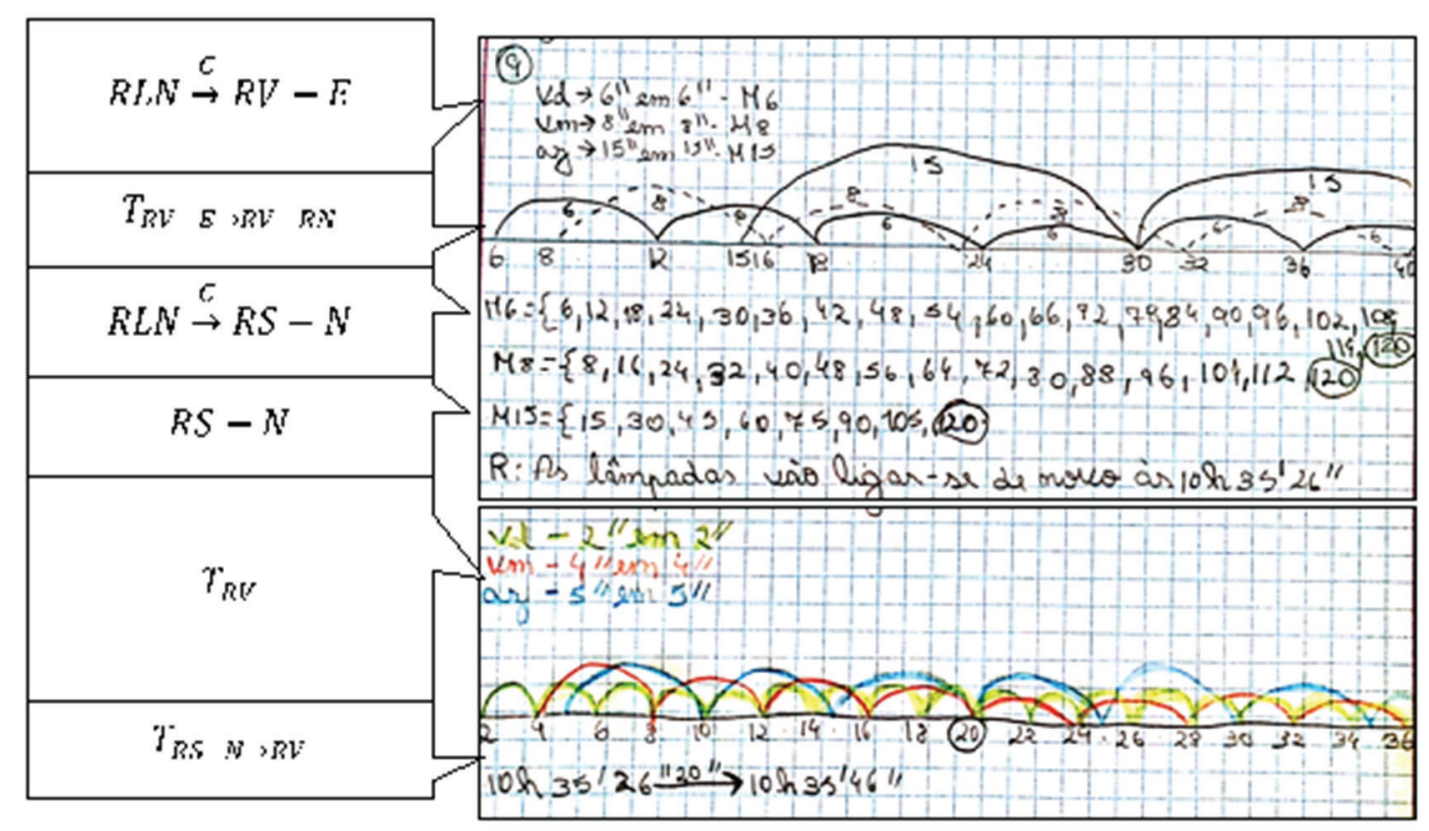


Nesse exemplo podem-se constatar tratamentos visuais a uma representação simbólica e a uma representação visual, e conversões (i) de linguagem verbal (enunciado do problema) para visual (reta numérica); (ii) de linguagem verbal para linguagem simbólica.

\section{Resultantes do Teste}

A Tabela 3 resume os resultados dos alunos, e as representações usadas no teste, nos dois momentos de aplicação.

Tabela 3 - Resultados e representações do teste nos dois momentos de aplicação.

\begin{tabular}{|c|c|c|c|c|}
\hline \multirow{2}{*}{ Item } & \multicolumn{2}{|r|}{ Teste (fim de novembro) } & \multicolumn{2}{|r|}{ Teste (fim de fevereiro) } \\
\hline & & Representações & & Representações \\
\hline 1 & (11) 65 & RS-N & (10) 59 & RS-N \\
\hline 2 & (12) 71 & RS-N; RLN; RV-F; RLN & (12) 71 & RS-N; RLN; RV-F; RLN \\
\hline 3 & (11) 65 & RV-DV; RS-N; RV-E & (8) 47 & RV-DV; RS-N \\
\hline 4 & (8) 47 & RS-N; RV-DV; RV-RN & (11) 65 & RS-N; RV-DV; RV-RN \\
\hline 5 & (6) 35 & RLN; RS-N; RV-DV; RV-E & (7) 41 & RLN; RS-N; RV-DV \\
\hline 6 & (6) 35 & RV-F; RV-S; RLN; RS-N; RV-DV & (10) 59 & RV-F; RV-S; RLN; RS-N; RV-DV \\
\hline 7 & (11) 65 & RV-E; RV-RN; RS-N; RV-F & (10) 59 & RV-S; RV-RN; RS-N; RV-F \\
\hline
\end{tabular}

Nota: Os quantitativos se referem à categoria de análise das respostas corretas do teste. A diferença para o total $(17[100 \%])$, que não se apresenta, representa as respostas erradas e as ausências de resposta, às quais nos referimos no texto seguinte.

De uma maneira geral todos os alunos utilizaram representações em diferentes sistemas de representação ao longo de todo o teste, tanto no primeiro como no segundo momento de aplicação. Verificou-se, no entanto, que a representação mais usada foi a representação numérica (linguagem simbólica) sendo essa representação exclusivamente usada na resposta ao primeiro item, nos dois momentos de aplicação.

Nas respostas ao segundo item (ver Figura 1), uma das representações sempre presente foi a representação numérica (RS-N), que fez par com uma representação visual (RV-F), com outra simbólica (RS-N) ou com nenhuma. Constatou-se um uso indevido do diagrama (RV-DV) para representar os múltiplos de cinco, mas tal não impediu o sucesso na resposta. Paralelamente, verificou-se um uso incorreto da mesma representação visual em algumas respostas ao item 4 , mas, também aqui, o seu uso indevido não influenciou negativamente o sucesso.

Foi na resposta ao item 3 que os alunos manifestaram o pior desempenho, tendo piorado da primeira aplicação para a segunda. Esses alunos fizeram, na sua maioria, a conversão de uma representação visual para uma representação numérica. 
A linguagem natural foi a representação escolhida pela maioria dos alunos para responder ao item 5, no entanto houve respostas com o uso de uma de duas representações de sistemas diferentes: a representação numérica (SR-N) ou a representação visual (RV-DV) para determinar os múltiplos comuns dos dois números envolvidos, e a representação em linguagem natural (RLN) para justificação do resultado.

No que diz respeito ao desempenho na resolução de problemas, houve uma melhoria (de seis para dez respostas corretas) no primeiro (envolvendo o m.d.c.) e, no segundo (envolvendo o m.m.c.) registrou-se que o desempenho piorou (de 11 para dez respostas corretas). Todos os alunos responderam a todas as questões do teste na primeira aplicação com exceção de um que não respondeu ao item 6 mas fê-lo corretamente na aplicação seguinte, com uma representação visual. Outro aluno não respondeu a duas questões do teste na segunda aplicação, tendo-o feito incorretamente na primeira.

Da análise global dos resultados da Tabela 3, constatou-se o uso de representações nos três sistemas de linguagem nas respostas ao Teste 1, que se manteve no Teste 2. Globalmente, os alunos participantes nessa intervenção de ensino melhoraram os resultados: $\mathrm{O}$ número de respostas corretas diminuiu em três itens ( 1 e 7 com menos uma resposta correta e o item 3 com menos 3 respostas corretas) manteve-se no item 2, e aumentou em três itens (o 4 com mais três respostas corretas, o 5 com mais uma resposta correta e o 6 com mais quatro respostas corretas).

\section{DisCuSSÃo E CONCLUSÃo}

Relembramos a questão que norteou este estudo: Qual o impacto da introdução de representações visuais, e suas transformações, na resolução de problemas com múltiplos e divisores? Os resultados mostraram que as representações visuais permitem transformações de representações como as representações simbólicas e as verbais (exemplos nas Figuras 2 e 3 e na Tabela 2. A introdução de representações visuais proporcionou um ambiente de aprendizagem com representações múltiplas (exemplos nas Figuras 2 e 3), o que, de acordo com Ainsworth (2008) e Duval (2006a, 2006b), proporciona um ambiente de compreensão dos conceitos matemáticos envolvidos e, de acordo com Dienes (1973), promove a abstração. Também constatou-se que a introdução frequente de representações visuais aumentou o leque de transformações de representações, tanto tratamentos como conversões (Tabela 3), tendo-se verificado uma maior variedade de representações na resolução de problemas e esse efeito prolongou-se no intervalo de tempo considerado. No entanto, também constataram-se erros na escolha da representação visual adequada à situação, o que sugere dificuldades não só no conceito mas também na representação, confirmando o trabalho de Van Garderen (2006), e a necessidade de regras (Ainsworth, 2008), bem como a pertinência de mais investigação nessa área.

De acordo com os nossos resultados concluímos que a utilização frequente de representações visuais potencia as transformações de representações, tanto tratamentos como conversões. A transformação de representações permitida e potenciada pela introdução das representações visuais teve como consequência a diversificação dos meios disponíveis pelos alunos na 
resolução desses problemas com influência no tempo de retenção desses assuntos na memória dos aprendentes, o que aumentou o seu êxito dentro do intervalo de tempo considerado.

\section{REFERÊNCIAS}

AINSWORTH, S. The educational value of multiple-representations when learning complex scientific concepts. In J. K. Gilbert (Orgs.); Visualization: Theory and practice in science education (p. 191-208). Dordrecht, The Netherlands: Springer, 2008.

ARCAVI, A. The role of visual representations in the learning of mathematics. Educational Studies in Mathematics, 52, 215-241, 2003.

BARBOSA, A. A resolução de problemas que envolvem a generalização de padrões em contextos visuais: um estudo longitudinal com alunos do 2. ${ }^{\circ}$ Ciclo do ensino básico. Braga: [s.n.], 2010. Tese de doutoramento, 2010.

Disponível em https://repositorium.sdum.uminho.pt/bitstream/1822/10561/1/tese.pdf

BRANSFORD, J. D., \& SCHWARTZ, D. L. Rethinking Transfer: A Simple Proposal with Multiple Implications. Review of Research in Education, 24, 61-100, 1999.

CALADO, I. A utilização educativa das imagens. Porto: Porto Editora, 1995.

COHEN, L., MANION, L., \& MORRISON K. Research methods in education. NY: Routledge, 2007.

DIENES, Z. The six stages in the process of learning mathematics. Slough: NFER-Nelson, 1973.

DREYFUS, T. On the status of visual reasoning in mathematics and mathematics education. In F. Furinghetti (Org.). Proceedings of the 15th Conference of the International Group for the Psychology of Mathematics Education (PME), 1, (p.33-48). Assisi, Italy : Università di Genova, 1991.

DUVAL, R. A cognitive analysis of problems of comprehension in a learning of mathematics. Educational Studies in Mathematics, 61 (1-2), 103-131, $2006 \mathrm{a}$.

Quelle sémiotique pour l'analyse de l'activité et des productions mathématiques? Relime, 9 (1), 45-82, $2006 \mathrm{~b}$.

EL MOUHAYAR, R., \& JURDAK, M. Teachers' ability to identify and explain students' actions in near and far figural pattern generalization tasks. Educational Studies in Mathematics, 82, 379-396, 2013.

GOLDIN, G. A. Perspectives on representation in mathematical learning and problem solving. In L. English (Org.), Handbook of international research in mathematics education (p. 178-203), New York, NY: Routledge, 2008.

LUÍS, F.. Em busca da solução: Relação entre a capacidade de resolução de problemas e o sucesso escolar em alunos dos $2 .^{\circ}$ e $3 .^{\circ}$ Ciclos do Ensino Básico. Tese de doutoramento, Universidade de Coimbra, 2015. Disponível em http://hdl.handle.net/10316/28376. 
MEC. Programa e metas curriculares Matemática Ensino Básico, Lisboa, Portugal: MEC, 2013.

NCTM. Principle and standards for school mathematics. Reston, VA: NCTM, 2000.

PRESMEG, $N$. Research on visualization in learning and teaching mathematics: Emergence from psychology. In: A. Gutiérrez \& P. Boero (Orgs.). Handbook of research on the psychology of mathematics education (p. 205-235). Dordrecht, Netherlands: Sense Publishers, 2006.

RYVE, A., NILSSON, P., \& PETTERSSON, K. Analyzing effective communication in mathematics group work: The role of visual mediators and technical terms. Educational Studies in Mathematics, 82, 497-514, 2013.

SEDIG, K., \& SUMNER, M. Characterizing interaction with visual mathematical representations. International Journal of Computers for Mathematical Learning, 11(1), 1-55, 2006.

SPIRO, R. J. Cognitive Flexibility Theory: Advanced Knowledge Acquisition in IllStructured Domains. Technical Report. 441, 1988.

STYLIANOU, D. A., \& SILVER, E. A. The role of visual representations in advanced mathematical problem solving: An examination of expert-novice similarities and differences. Mathematical Thinking and Learning, 6(4), 353-387, 2004.

VAN GARDEREN, D. Teaching Visual Representation for mathematics problem solving. In Montague, M., \& Jitendra, A. K. (Orgs.), Teaching mathematics to middle school students with learning difficulties, 2, (pp.72-88). New York: Guilford Press, 2006.

Este trabalho é financiado por Fundos Nacionais através da FCT - Fundação para a Ciência e a Tecnologia no âmbito do projeto UID/CED/00194/2013.

\section{Dados dos Autores}

Paula Montenegro

Universidade de Trás-os-Montes e Alto Douro. Vila Real - Portugal. mpmvcardoso@ gmail.com

Cecília Costa

Departamento de Matemática, Universidade de Trás-os-Montes e Alto Douro. Vila Real - Portugal. Centro de Investigação Didática e Tecnologia na Formação de Formadores. mcosta@utad.pt

BERNARDINO LOPES

Departamento de Física, Universidade de Trás-os-Montes e Alto Douro. Vila Real - Portugal. Centro de Investigação Didática e Tecnologia na Formação de Formadores. blopes@ utad.pt

Submetido em: 16-12-2016

Aceito em: 20-2-2017

68 Comunicações |Piracicaba | v. 24 | 1 . 1 | p. 55-68 | janeiro-abril 2017 